\title{
An additional broad line region in 3C 390.3 associated with the jet
}

\author{
L. S. Nazarova ${ }^{1}$ and N. G. Bochkarev ${ }^{2}$ \\ ${ }^{1}$ EAAS, Universitetskij pr.13, 119992, Moscow, Russia \\ email:1snazarova@rambler.ru \\ ${ }^{2}$ SAI, Universitetskij pr 13, 119992, Moscow, Russia \\ email:boch@sai.msu.ru
}

\begin{abstract}
We suggest the existence of two BLRs in 3C 390.3 which have different locations. The BLR1 is located at a distance in accordance to the hydrogen line time lags of $\approx 20$ days. This disk-like region emits predominantly low ionization lines. The BLR2 forms around the radio-jet and is located at the distance corresponding to time lags of $\approx 40-80$ days. The BLR2 is responsible for most of the emission in UV lines. The L $\alpha$ line partly forms in BLR2 (40-60\%) and partly in BLR1.
\end{abstract}

Keywords. galaxies: Seyfert, double-peaked emission lines - modelling: broad line region

The double-peaked galaxy 3C 390.3 is the prototype of the class of AGNs showing complex broad-line profiles with displaced distributions and/or an anisotropic illumination of the broad-line region (BLR). The time lag in 3C 390.3 for optic lines small $(\approx 20$ days)(Dietrich et al. 1998$)$ compare to the lag in UV lines CIV and L $\alpha(\approx 37$ and 60 days respectively)(O'Brien et al. 1998). This is differ from other AGNs.

The observed profiles of the CIV, $\mathrm{L} \alpha, \mathrm{H} \beta$ and $\mathrm{H} \alpha$ lines have been divided into seven parts, the width of each part being equal to $2000 \mathrm{~km} \mathrm{~s}^{-1}$. The core of the lines is measured between -1000 and $+1000 \mathrm{~km} \mathrm{~s}^{-1}$. Thus, the blue and red wings have a width of \pm 7000 $\mathrm{km} \mathrm{s}^{-1}$. The difference of the line fluxes along of line profiles taken for $\mathrm{L} \alpha, \mathrm{CIV}, \mathrm{H} \beta$ and $\mathrm{H} \alpha$ lines show two clear components, one shortward which better seen in UV lines and one near to the line center. The $\mathrm{CIV} / \mathrm{L} \alpha$ ratio is low in the low-velocity regions of the line profile, but becomes higher in the blue wing, particularly when 3C 390.3 is more active. The observed $\mathrm{L} \alpha / \mathrm{H} \beta$ ratio is high at low velocity and decreases in the wings for both high and low states of the nuclear activity. The $\mathrm{H} \alpha / \mathrm{H} \beta$ ratio is higher at low velocity in both the minimum and the maximum of nuclear activity.

The broad line spectrum is computed with the photoionization cod "CLOUDY" which consist in shell of gas (or cloud) in ionization and thermal equilibrium under the action of an external flux of radiation illuminating one face. The comparison of the observed and the computed $\mathrm{CIV} / \mathrm{L} \alpha, \mathrm{L} \alpha / \mathrm{H} \beta$ and $\mathrm{H} \alpha / \mathrm{H} \beta$ line ratios supports the suggestion that there are two different emission regions with a different densities :

- a low-density region with density: $10^{8-9} \mathrm{~cm}^{-3}$ located at the distance 40-80 days from the center (HIL zone);

- a high-density region with the density $10^{10-12} \mathrm{~cm}^{-3}$ or more located at the distances $\approx 20$ days (LIL zone).

The contribution from the LIL zone with the high electron density $\mathrm{N}_{e} \approx 10^{10-12} \mathrm{~cm}^{-3}$ should be significant at the wings where $\mathrm{L} \alpha / \mathrm{H} \beta \approx 2$. However the emission from HIL zone could predicts $40-60 \%$ contribution of the variable part $\mathrm{L} \alpha / \mathrm{H} \beta \approx 7,42$.

Arshakian et. al. (2010) found a significant correlation between an ejected jet components and optical continuum flares. It is pointed out that the emission in the optical 

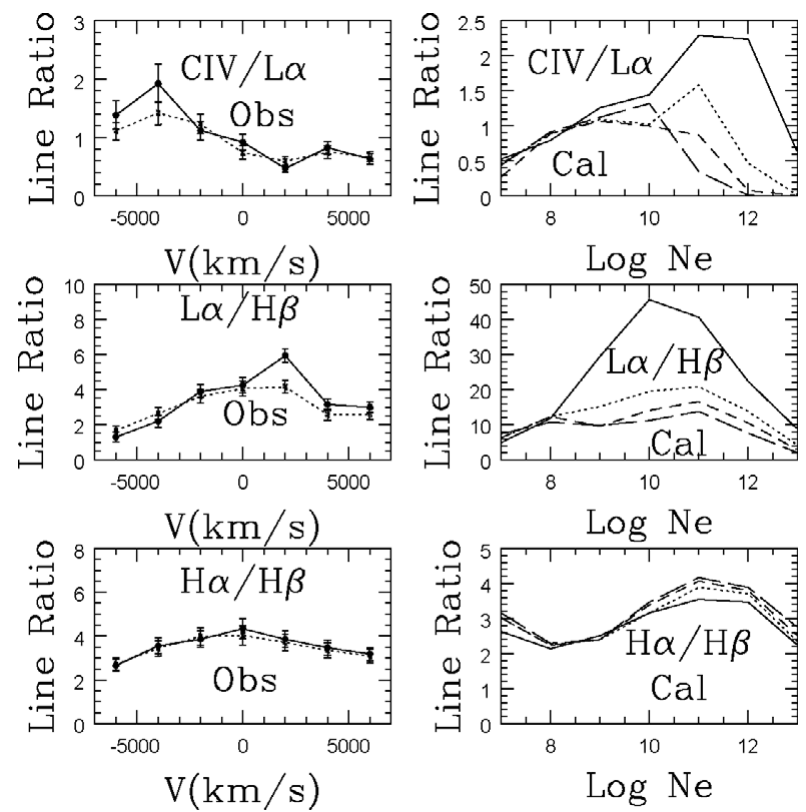

Figure 1. Line ratios $\mathrm{CIV} / \mathrm{L} \alpha, \mathrm{L} \alpha / \mathrm{H} \beta$ and $\mathrm{H} \alpha / \mathrm{H} \beta$ for the maximum states of nuclear activity. The observed line ratios - left side; the calculated line ratios - right side

lines might exist not only at the disk but also near to the radio-jet. According to the observed lags for Balmer and UV lines obtained of AGN Watch campaign and also taking into account our modeling we suggest the existing of two BLRs in 3C390.3 which have a different locations.

The BLR1 traditionally located at the disk at the distance according to the hydrogen lines $\approx 20$ days. This disk-like region emits predominantly low ionization lines located in the equatorial plane where the BLR covering approaches 100\%. The BLR2 forms around of the radio-jet and located at the distance $\approx 40-80$ days from the center. The BLR2 responsible for the most emission in the UV lines (CIV line is varied at $84 \%$ ). The L $\alpha$ line partly forms in BLR2 - 40-60\% and partly in BLR1. The bulk of the hydrogen lines forms at the disk and the wings - near to the radio-jet at the distance from the center $\geqslant 80$ days.

\section{References}

Arshakian T. G., Leon-Tavares J., Lobanov A. P., Chavushyan V. H., Shapovalova A. I., Burenkov, A. N., \& Zensus J. A. 2010, MNRAS 401, 1231A

Deitrich, M., Peterson, B. M., Albrecht, P., Altmann, M., Barth, A. J., and 53 coauthors 1998, ApJS 115, 185D

O'Brien, P. T., Dietrich, M., Leight, K., Alloin, D., Clavel, J., Grenshaw, D. M., and 41 coauthors 1998, ApJ 509, 1630 